\title{
Alteration of Gut Microbiota in Carbapenem-Resistant Enterobacteriaceae Carriers during Fecal Microbiota Transplantation According to Decolonization Periods
}

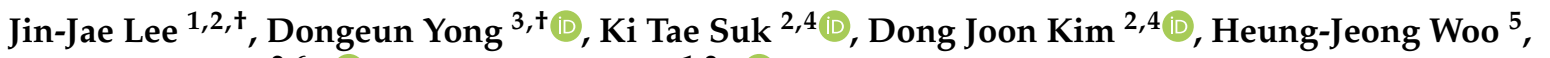 \\ Seung Soon Lee ${ }^{2,6, *(\mathbb{D})}$ and Bong-Soo Kim ${ }^{1,2, * \text { (D) }}$
}

check for

updates

Citation: Lee, J.-J.; Yong, D.; Suk, K.T.; Kim, D.J.; Woo, H.-J.; Lee, S.S.; Kim, B.-S. Alteration of Gut

Microbiota in Carbapenem-Resistant Enterobacteriaceae Carriers during Fecal Microbiota Transplantation According to Decolonization Periods. Microorganisms 2021, 9, 352. https:// doi.org/10.3390/microorganisms 9020352

Academic Editor: Giorgos Bamias

Received: 7 January 2021

Accepted: 8 February 2021

Published: 10 February 2021

Publisher's Note: MDPI stays neutral with regard to jurisdictional claims in published maps and institutional affiliations.

\section{Copyright: (c) 2021 by the authors.} Licensee MDPI, Basel, Switzerland. This article is an open access article distributed under the terms and conditions of the Creative Commons Attribution (CC BY) license (https:// creativecommons.org/licenses/by/ $4.0 /)$.
1 Department of Life Science and Multidisciplinary Genome Institute, Hallym University, Chuncheon 24252, Korea; lij807@hallym.ac.kr

2 Institute for Liver and Digestive Diseases, Hallym University, Chuncheon 24252, Korea; ktsuk@hallym.ac.kr (K.T.S.); djkim@hallym.ac.kr (D.J.K.)

3 Department of Laboratory Medicine and Research Institute of Bacterial Resistance, Yonsei University College of Medicine, Seoul 03722, Korea; DEYONG@yuhs.ac

4 Department of Internal Medicine, Division of Gastroenterology and Hepatology, Hallym University, Chuncheon Sacred Heart Hospital, Chuncheon 24253, Korea

5 Department of Internal Medicine, Division of Infectious Diseases, Hallym University Dongtan Sacred Heart Hospital, Hallym University College of Medicine, Hwaseong 18450, Korea; infwoo@hallym.or.kr

6 Department of Internal Medicine, Division of Infectious Diseases, Hallym University Chuncheon Sacred Heart Hospital, Hallym University College of Medicine, Chuncheon 24252, Korea

* Correspondence: hushh93@gmail.com (S.S.L.); bkim79@hallym.ac.kr (B.-S.K.)

+ Equal contribution.

\begin{abstract}
Fecal microbiota transplantation (FMT) has been suggested as an alternative therapeutic option to decolonize carbapenem-resistant Enterobacteriaceae (CRE). However, the analysis of gut microbiota alteration in CRE carriers during FMT is still limited. Here, gut microbiota changes in CRE carriers were evaluated during FMT according to decolonization periods. The decolonization of 10 CRE carriers was evaluated after FMT, using serial consecutive rectal swab cultures. Alterations of gut microbiota before and after FMT (56 serial samples) were analyzed using high-throughput sequencing. The decolonization rates of CRE carriers were $40 \%, 50 \%$, and $90 \%$ within 1,3 and 5 months after initial FMT, respectively. Gut microbiota significantly changed after FMT ( $p=0.003)$. Microbiota alteration was different between the early decolonization carriers (EDC) and late decolonization carriers (LDC). Microbiota convergence in carriers to donors was detected in EDC within 4 weeks, and keystone genera within the Bacteroidetes were found in the gut microbiota of EDC before FMT. The relative abundance of Klebsiella was lower in EDC than in LDC, before and after FMT. Our results indicate that FMT is a potential option for CRE decolonization. The gut microbiota of CRE carriers could be used to predict decolonization timing after FMT, and determine repeated FMT necessity.
\end{abstract}

Keywords: carbapenem-resistant Enterobacteriaceae (CRE); fecal microbiota transplantation (FMT); gut microbiota; decolonization

\section{Introduction}

Carbapenem-resistant Enterobacteriaceae (CRE) is an urgent antibiotic threat due to high infection-associated mortality, limited therapeutic options, and potential to rapidly spread between bacterial species [1,2]. In the healthcare setting, the carriage rates of extended spectrum beta-lactamase (ESBL) or CRE were $75.2 \%$ at 3 months, 55.3\% at 6 months, and $35.2 \%$ at 12 months, after initial identification [3]. Although short-term antibiotic therapy for the decolonization of antibiotic-resistant bacteria (ARB) could reduce carriage during therapy, its long-term effects are unclear [3]. Pathogens can acquire resistance to antibiotics, and side effects of the antibiotics on gut microbiota, including increased susceptibility to 
a range of infections through inevitable microbiota dysbiosis, have been reported $[4,5]$. Therefore, alternative solutions to reduce or eradicate ARB in patients are necessary.

Gut microbiota plays important roles in metabolic processes, immune modulation, and protection against pathogen colonization. As microbiota dysbiosis can be linked to function loss, microbiota engraftment from healthy donors can recover these functions. Fecal microbiota transplantation (FMT) has become a popular approach to microbiota modulation and has been widely used as a treatment option for multiple recurrences of Clostridioides difficile infection (CDI), with a substantial reduction in antibiotic resistance genes as well as gut microbiota changes [6,7]. A high response rate to FMT in CDI treatment has led to applications in various diseases, including ulcerative colitis, Crohn's disease, and multiple antibiotic resistance [8-10]. The prevalence of Enterobacteriaceae with multiple resistance to beta-lactam antibiotics, including carbapenems, vancomycin-resistant Enterococcus (VRE) and methicillin-resistant Staphylococcus aureus (MRSA), could be reduced by FMT [11]. FMT's efficacy in decolonizing multidrug-resistant (MDR) organisms and preventing recurrent MDR infections was 37.5 to $87.5 \%$ in a previous study [10]. CRE carriers showed decreased diversity in gut microbiota and increased relative abundance of Enterobacteriaceae [12]. Therefore, FMT could be an attractive option to reduce CRE by gut microbiota modulation. However, the analysis of gut microbiota alteration in CRE carriers during FMT is still limited.

To understand the influence of indigenous gut microbiota on the decolonization of CRE and the alteration of microbiota during FMT, we analyzed the longitudinal changes in gut microbiota from 10 carbapenemase-producing (CP-) CRE carriers during FMT treatment over the stipulated decolonization periods.

\section{Materials and Methods}

\subsection{Subject Selection and FMT Procedures}

CP-CRE carriers ( $\geq 18$ years old) with one or more of the following risk factors for prolonged carriage of CP-CRE were selected for FMT treatment at Hallym University Chuncheon Sacred Heart Hospital: (1) Carbapenem use for $>3 \mathrm{~d}$ after CP-CRE identification; (2) Positive CP-CRE in clinical specimens, defined as every clinical specimen positive for CP-CRE other than rectal swab, regardless of clinical signs of infection; (3) The presence of concurrent CDI after CP-CRE identification; (4) Long duration of hospitalization ( $>2$ months) after CP-CRE acquisition [13]. Prolonged carriage of CP-CRE was defined as the identification of CRE in either a rectal swab or clinical culture for $>3$ months after initial detection.

Frozen or capsulized gastrointestinal microbiota (MicroBiotix, Inc., Seoul, Korea) from unrelated pre-screened healthy donors were supplied on the day of each FMT after informed consent was provided. The human microbiota banking project of the Microbiotix Corporation, a non-profit human microbiota bank for FMT, was approved by the Severance Hospital Institutional Review Board, Seoul, Korea (IRB no. 4-2016-0871). Screening tests for donors were performed in a two-stage process based on the Korean Transfusion Guidelines and the European and American human microbiota banks' donor screening protocols [14-17]. Firstly, an in-person clinical assessment was performed to evaluate the general health and gastrointestinal conditions of donor candidates, as well as any risk factors for transmissible diseases. Secondly, screening for viral, bacterial, and protozoal pathogens, serological/stool screening, a urea breath test, and a chest posteroanterior radiography were performed to exclude donors with potentially transmissible pathogens.

Antibiotic administration was stopped at least $48 \mathrm{~h}$ before performing FMT. A bowel lavage was performed a day prior to FMT. A frozen fecal microbiota preparation was thawed in a water bath at $30^{\circ} \mathrm{C}$ for $2 \mathrm{~h}$ prior to FMT. Thawed FMT preparations were divided into five $50 \mathrm{~mL}$ syringes for administration via a colonoscopy or esophagogastroduodenoscopy. The FMT was mainly performed at the distal end of the ileum or ascending colon via a colonoscopy. If bowel preparation was not performed correctly or entry of the colonoscope was not smooth, FMT was performed at the duodenum by switching 
to esophagogastroduodenoscopy. Capsulized FMT was performed on one patient who failed to decolonize CRE, even in the second FMT. Twenty pills of capsulized stool were administered to the patient along with cranberry juice daily, for 2 consecutive days. Rectal swabs were collected before FMT and once a week after FMT treatment and stored at $-80{ }^{\circ} \mathrm{C}$ until further use.

Decolonization of CP-CRE was defined as three consecutive CRE-negative rectal swab cultures repeated with a $3 \mathrm{~d}$ interval after FMT. The decolonization time of CP-CRE after FMT was defined as the time interval from the initial time point of FMT until the time point of three consecutive CRE-negative surveillance rectal swab cultures, as well as clinical cultures. The study protocol was approved by the Institutional Review Board at Hallym University Chuncheon Sacred Heart Hospital (IRB no. 2019-05-012 and 202008-006) and was registered on the ClinicalTrials.gov public website (no. NCT04583098). Written informed consent for participation in this study was obtained from all patients. The study complies with the Declaration of Helsinki.

\subsection{Culture Assay and Polymerase Chain Reaction (PCR) for CRE Detection in Carriers}

Specimens including rectal swabs were collected from patients during the study period. Rectal swabs were plated on primary CHROMagar KPC medium (CHROMagar, Paris, France) for CRE screening. Other clinical specimens such as sputum, urine, and blood were plated on blood agar (Becton-Dickinson, Sparks, MD, USA) and MacConkey agar (BectonDickinson). Agar plates were incubated at $36.5^{\circ} \mathrm{C}$ for $16-24 \mathrm{~h}$. Taxonomic identification and antibiotic susceptibility tests, including carbapenem for isolates, were performed using the VITEK ${ }^{\circledR} 2$ system (bioMerieux, Marcy I'Etoile, France) according to the Clinical and Laboratory Standards Institute M100S guidelines [18]. Carbapenemase production in each isolate was analyzed using the CarbaNP test [19]. To validate carbapenamase production in isolates, target genes encoding $b l a_{\mathrm{KPC}}, b l a_{\mathrm{NDM}}, b l a_{\mathrm{IMP}}, b l a_{\mathrm{VIM}}$, and $b l a_{\mathrm{OXA}-48}$ were amplified and confirmed by gel electrophoresis [20].

\subsection{Quantitative Real-Time PCR and MiSeq Sequencing}

Metagenomic DNA was extracted from collected rectal swab samples using the RNeasy PowerMicrobiome RNA Isolation Kit (Qiagen, Hilden, Germany) according to the manufacturer's instructions. Extracted DNA was verified using 1\% agarose gel electrophoresis, and quantification was performed using the BioPhotometer D30 and $\mu$ Cuvette G1.0 (Eppendrof, Hamburg, Germany).

The $16 \mathrm{~S}$ rRNA gene copies of bacteria in each sample were measured and compared by quantitative real-time PCR, based on the 16S rRNA gene using 340F (5'-TCCTACGGGAGG CAGCAG-3') and 518R (5'-ATTACCGCGGCTGCTGG-3') primers on a Thermal Cycler Dice Real Time System III (Takara Bio). Amplifications were conducted in a final volume of $25 \mu \mathrm{L}$, containing $12.5 \mu \mathrm{L} 2 \times$ SYBR green premix Ex Taq (Takara Bio, Otsu, Japan), $2 \mu \mathrm{M}$ of each primer, and $1 \mu \mathrm{L}$ of DNA template (10-fold serial dilution of DNA) or distilled water (negative control). The reaction conditions consisted of an initial denaturation at $95{ }^{\circ} \mathrm{C}$ for $5 \mathrm{~min}$, followed by $40 \mathrm{cycles}$ of $95^{\circ} \mathrm{C}$ for $5 \mathrm{~s}$ and $60^{\circ} \mathrm{C}$ for $30 \mathrm{~s}$. Standard curves were generated from parallel reactions with serial log-concentrations of the copy number of the bacterial 16S rRNA gene from Escherichia coli K12 W3110, and each reaction was performed in triplicate.

To analyze the microbiota in samples, metagenomic DNA was amplified following the 16S Metagenomic Sequencing library for the MiSeq system manufacturer's guidelines (Illumina, San Diego, CA, USA), as described previously [21,22]. Briefly, the V1-V3 hypervariable regions of the $16 \mathrm{~S}$ rRNA gene were amplified using primers with adapters (forward: $5^{\prime}$-adapter [TCGTCGGCAGCGTCAGATGTGTATAAGAGACAG]- GAGTTTGATCMTGG CTCAG-3'; reverse: 5'-adapter [GTCTCGTGGGCTCGGAGATGTGTATAAGAGACAG]ATTACCGCGG CTGCTGG-3'). The first step of amplification was performed in a final volume of $25 \mathrm{uL}$ containing $1 \mu \mathrm{M}$ of each primer, $1.25 \mathrm{U}$ Ex Taq polymerase (Takara Bio), $2.5 \mu \mathrm{L}$ Ex Taq Buffer $(10 \times), 4 \mu \mathrm{L}$ dNTP Mix, and $2.5 \mu \mathrm{L}$ DNA template. Initial denaturation 
was conducted at $95^{\circ} \mathrm{C}$ for $3 \mathrm{~min}$, followed by 25 cycles of denaturation at $95^{\circ} \mathrm{C}$ for $30 \mathrm{~s}$, annealing at $55{ }^{\circ} \mathrm{C}$ for $30 \mathrm{~s}$, extension at $72{ }^{\circ} \mathrm{C}$ for $30 \mathrm{~s}$, and final extension at $72{ }^{\circ} \mathrm{C}$ for $5 \mathrm{~min}$. The amplicons were verified with $2 \%$ agarose gel electrophoresis, and purification and size selection were performed using the Agencourt AMPure XP beads (Beckman Coulter, Indianapolis, IN, USA). Index PCR reactions were performed using $5 \mu \mathrm{L}$ purified PCR product in a final volume of $50 \mu \mathrm{L}$ using the Nextera XT Index Kit (Illumina). The index PCR reaction conditions consisted of initial denaturation at $95{ }^{\circ} \mathrm{C}$ for $3 \mathrm{~min}$, followed by 8 cycles of denaturation at $95^{\circ} \mathrm{C}$ for $30 \mathrm{~s}$, annealing at $55^{\circ} \mathrm{C}$ for $30 \mathrm{~s}$, extension at $72{ }^{\circ} \mathrm{C}$ for $30 \mathrm{~s}$, and final extension at $72{ }^{\circ} \mathrm{C}$ for $5 \mathrm{~min}$. The PCR products of each sample were purified again using Agencourt AMPure XP beads (Beckman Coulter). The library was quantified using the Takara PCR Thermal Cycler Dice Real Time System III with the GenNext NGS Library Quantification Kit (Toyobo, Osaka, Japan). To detect contamination in each experimental step, negative controls (a blank swab and distilled water) were performed with the same procedures, along with fecal samples. Equimolar concentrations of each library from different samples were pooled and sequenced on the Illumina MiSeq system (300 bp paired ends) according to the manufacturer's instructions.

\subsection{Sequencing Data Analysis}

The obtained sequence reads were analyzed using the DADA2 R package (ver 1.14.0) [23] and operational taxonomic units (OTUs)-based analyses. The DADA2 microbiome pipeline was used for pre-processing, including quality check, de-noising, merging, and chimera removal. OTUs-based analyses were further analyzed using the amplicon sequence variants (ASVs) table obtained after pre-processing. Briefly, sequence reads were quality-filtered and trimmed using the filterAndTrim() function; an expected error threshold of 2, combined with trimming $10 \mathrm{bp}$ from the ends of forward reads and $40 \mathrm{bp}$ from the ends of reverse reads, and short length sequences and ambiguous sequence bases were removed by default parameters of DADA2. Filtered reads were subsequently de-noised by sequencing error rates. A model for sequencing error rates was constructed using the learnErrors() function. ASVs were inferred from filtered sequences using the dada() function. ASVs from forward and reverse sequences were merged using the mergePairs() function, and chimeric ASVs were detected and removed using the removeBimeraDenove() function. The fasta file of each sample was extracted from non-chimeric ASVs and the resulting sequences were subsequently clustered into OTUs using a 97\% sequence similarity from the CLC Genome Workbench OTU clustering pipeline (ver. 8.5.1, Qiagen). Taxonomic classification of the OTUs was performed using the EzTaxon database [24]. To compare the diversity indices between samples, read numbers were normalized by random subsampling, and diversity indices were calculated using MOTHUR (ver. 1.35.1) [25]. Linear discriminant analysis effect size (LEfSe) was used to identify different genera between groups [26]. Genera with a logarithmic LDA score $>2.0$ were considered significantly different.

\subsection{Co-Occurrence Network Analysis}

Co-occurrence networks were constructed to explore the interaction patterns among different bacterial taxa, based on the CoNet module in Cytoscape (ver. 3.7.2) [27]. The 30 most abundant genera in each group were selected for the co-occurrence network. Four similarity matrices were calculated using the Pearson and Spearman correlation methods, mutual information similarity method, Bray-Curtis dissimilarity, and Kullback-Leibler dissimilarity distance methods to construct networks. The threshold edge number was set to 100. A bootstrap resampling method with 1000 iterations in an edge score routine was employed to achieve randomization, and a Benjamini-Hochberg multiple test correction (corrected $p<0.01$ ) was performed to remove false positives. $p$ value merging was achieved using the Brown method to obtain the final co-occurrence networks. Topological parameters were determined by NetworkAnalyzer (ver. 2.7), and keystone genera were selected based on closeness centrality and betweenness centrality values. 


\subsection{Statistical Analysis}

Significant differences between groups were calculated using the Mann-Whitney U test in R software (ver. 3.6.1). Non-metric multidimensional scaling (NMDS) was tested using analysis of similarities (ANOSIM) as implemented in the vegan R package. Results with $p<0.05$ or corrected $p<0.05$ were considered significant. Correlations between bacterial members were calculated by Spearman's rank correlation coefficient in R software. Indicator genera $(p<0.05)$ were determined using the labdsv R package.

\section{Results}

\subsection{Clinical Features of CP-CRE Carriers}

FMT was performed to decolonize the CRE in 10 carriers with the risk of prolonged CP-CRE carriage, and their baseline characteristics are shown in Table 1. The mean age of carriers was $72.7 \pm 7.8$ years, and seven carriers were female. The average duration of CP-CRE carriage prior to FMT was $5.7 \pm 2.1$ months. The decolonization rates were $40.0 \%$ $(4 / 10), 50.0 \%(5 / 10)$, and $90.0 \%(9 / 10)$ within 1,3 , and 5 months after initial FMT treatment, respectively. The average and median time to successful decolonization after initial FMT were $65.2 \pm 4.9 \mathrm{~d}$ and $51.0 \mathrm{~d}$, respectively. Despite having risk factors for prolonged CP-CRE carriage, four carriers (40\%) showed faster successful decolonization within 1 month after initial FMT than the other carriers. Three carriers showed decolonization after the second FMT, and one carrier showed decolonization after the third FMT treatment. One carrier (no. 7) was not decolonized for $138 \mathrm{~d}$ despite the second FMT. The overall decolonization rate was $90.0 \%$ (9/10), irrespective of the time point after FMT treatment.

\subsection{Changes in Gut Microbiota of CP-CRE Carriers after FMT Treatment}

CRE decolonization and changes in gut microbiota after FMT were analyzed by both culture assays and $16 \mathrm{~S}$ rRNA-based sequencing. The sampling times and decolonization day in each carrier are indicated in Figure S1. The detection of carbapenem-resistant Enterobacteriaceae by culture during FMT is summarized in Supplementary Table S1. Nine carriers fulfilled the CRE decolonization criteria, and each patient had a different decolonization period.

Gut microbiota changes were analyzed in non-metric multidimensional scaling (NMDS) plots based on the Bray-Curtis dissimilarity, and gut microbiota significantly changed after FMT treatment (ANOSIM, $p=0.003$; Figure 1a). The diversity of gut microbiota in recipients increased to a greater extent after FMT than before FMT $(p<0.05$; Figure 1b). The differences of gut microbiota in carriers after FMT were unaffected by age and sex (ANOSIM, Age: $p=0.308$; Sex: $p=0.366$; Figure S2A), whereas they were significantly related to the follow-up sampling times after FMT treatment ( $p=0.041$; Figure S2B).

The relative abundance of Bacteroidetes significantly increased in the gut microbiota of all carriers after FMT treatment $(p<0.001$; Figure 1c). Other phyla were otherwise changed among carriers. Genera changes were compared via times after FMT (Figure S3). Although the changed genera were different in terms of times after FMT in each carrier, more diverse genera were detected after FMT than before FMT. The relative abundances of the CRE genera, Klebsiella and Escherichia, were compared along times in each carrier (Figure 1d). Although their changes fluctuated in terms of follow-up times, the relative abundances of Klebsiella were reduced after FMT. However, the proportions of Escherichia were maintained in some carriers. These results were consistent with those of culture assays (Table S1). 
Table 1. Summary of Characteristics of Selected CRE Carriers.

\begin{tabular}{|c|c|c|c|c|c|c|c|c|c|c|c|}
\hline Carrier No. & Age & Sex & CRE Type & $\begin{array}{c}\text { Duration of CRE } \\
\text { Carriage before FMT }\end{array}$ & $\begin{array}{l}\text { Carbapenem Use } \geq 3 \text { days } \\
\text { after CRE Carriage }\end{array}$ & $\begin{array}{l}\text { CRE () in Clinical Specimen } \\
\text { after CRE Carriage }\end{array}$ & $\begin{array}{c}\text { Concurrent C. Cifficile } \\
\text { Infection after CRE Carriage }\end{array}$ & $\begin{array}{l}\text { Prolonged Hospitalization } \geq \\
2 \text { Months after CRE Carriage }\end{array}$ & FMT Material & FMT Procedure & $\begin{array}{l}\text { Time to Decolonization of CRE } \\
\text { from FMT }\end{array}$ \\
\hline 1 & 78 & Female & KPC-CRE & 7 months & + & + & - & + & $\begin{array}{l}\text { Unrelated donor, } \\
\text { frozen stool }\end{array}$ & $\begin{array}{l}\text { Terminal ileum and ascending colon } \\
\text { via colonoscopy }\end{array}$ & 25 days \\
\hline 2 & 68 & Male & KPC-CRE & 4 months & + & + & - & + & $\begin{array}{l}\text { Unrelated donor, } \\
\text { frozen stool }\end{array}$ & $\begin{array}{l}\text { Duodenum via EGD and ascending } \\
\text { colon via colonoscopy }\end{array}$ & 26 days \\
\hline 3 & 80 & Female & KPC-CRE & 7 months & + & + & - & + & $\begin{array}{l}\text { Unrelated donor, } \\
\text { frozen stool }\end{array}$ & Duodenum via EGD (1st and 2nd FMT) & $\begin{array}{l}15 \text { days after 2nd FMT (106 days } \\
\text { after 1st FMT) }\end{array}$ \\
\hline 4 & 79 & Male & KPC-CRE & 6 months & + & + & - & + & $\begin{array}{l}\text { Unrelated donor, } \\
\text { frozen stool }\end{array}$ & Terminal ileum via colonoscopy & 51 days \\
\hline 5 & 75 & Female & KPC-CRE (\& VRE) & 5 months & + & + & + & + & $\begin{array}{l}\text { Unrelated donor, } \\
\text { frozen stool }\end{array}$ & Ascending colon via colonoscopy & 15 days \\
\hline 7 & 57 & Male & KPC-CRE & 7 months & + & - & - & + & $\begin{array}{l}\text { Unrelated donor, } \\
\text { frozen stool }\end{array}$ & $\begin{array}{l}\text { Terminal ileum and ascending colon via } \\
\text { colonoscopy (1st and 2nd FMT) }\end{array}$ & $\begin{array}{l}\text { not decolonized (followed until } \\
\text { 138 days after 1st FMT) }\end{array}$ \\
\hline 8 & 81 & Female & KPC-CRE & 10 months & + & + & + & + & $\begin{array}{l}\text { Unrelated donor, } \\
\text { frozen and } \\
\text { capsulized stool }\end{array}$ & $\begin{array}{l}\text { Duodenum via EGD (1st and } 2 \text { nd FMT), } \\
20 \text { capsules daily for } 2 \text { days (3rd FMT) }\end{array}$ & $\begin{array}{l}16 \text { days after 3rd FMT (137 days } \\
\text { after 1st FMT) }\end{array}$ \\
\hline 9 & 65 & Female & KPC-CRE & 3 months & + & - & - & + & $\begin{array}{l}\text { Unrelated donor, } \\
\text { frozen stool }\end{array}$ & $\begin{array}{l}\text { Terminal ileum via colonoscopy (1st) } \\
\text { FMT) duodenum via } \mathrm{ECD} \text { (2nd FMT) }\end{array}$ & $\begin{array}{l}16 \text { days after 2nd FMT (92 days } \\
\text { after 1st FMT) }\end{array}$ \\
\hline 10 & 69 & Female & KPC-CRE & 4 months & + & + & - & + & $\begin{array}{l}\text { Unrelated donor, } \\
\text { frozen stool }\end{array}$ & $\begin{array}{l}\text { Terminal ileum and ascending colon } \\
\text { via colonoscopy }\end{array}$ & 18 days \\
\hline
\end{tabular}


(a)

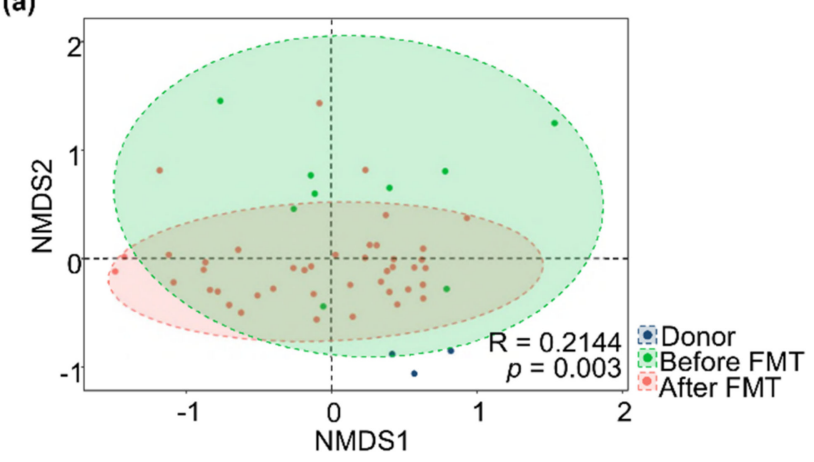

(b)

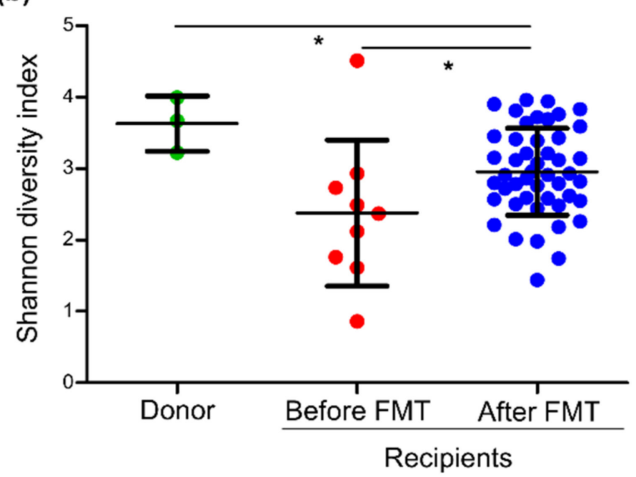

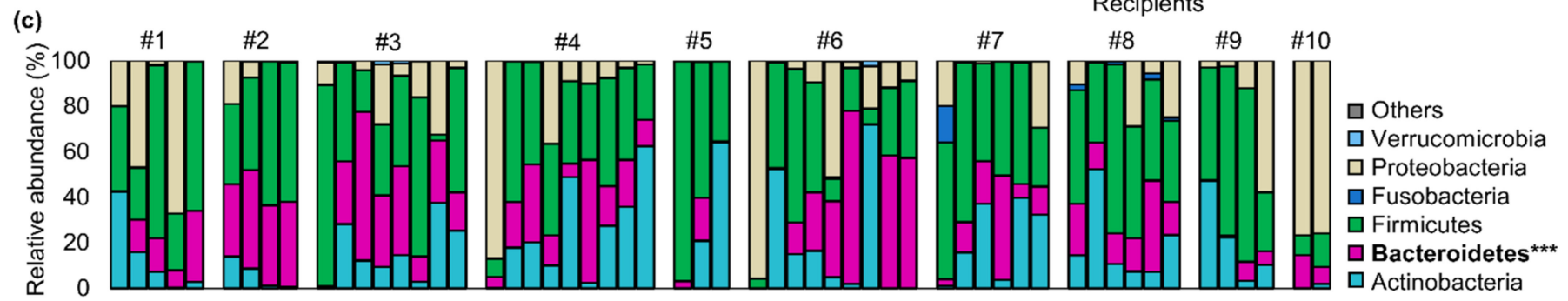

(d)

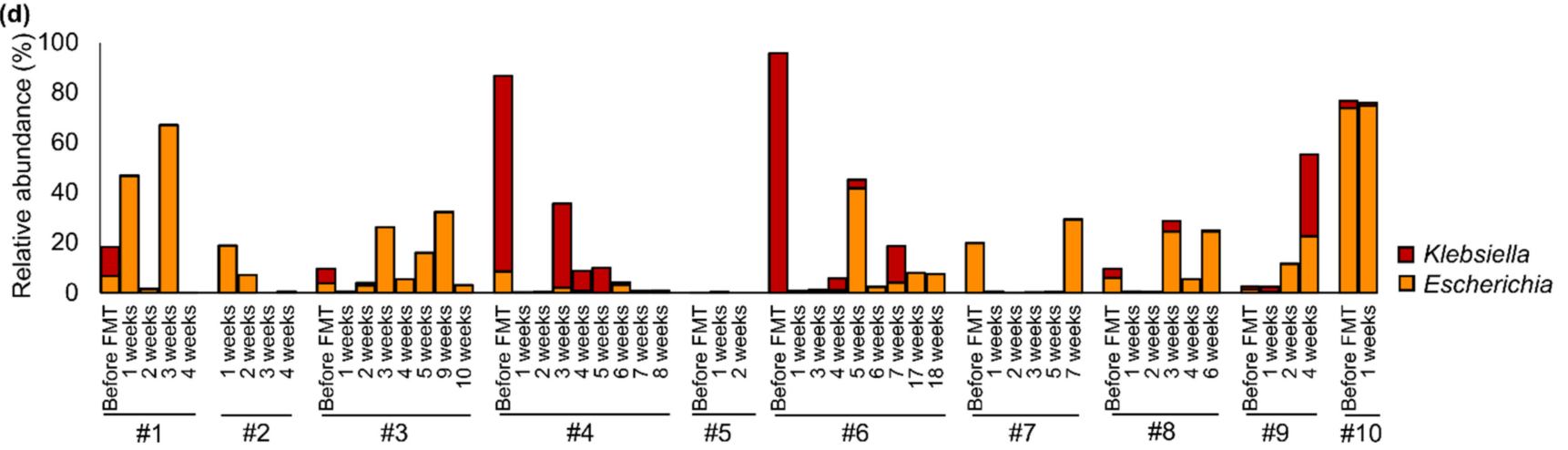

Figure 1. Gut microbiota changes in CRE carriers during FMT treatment. (a) The difference in microbiota between carriers and donors were compared in NMDS plots. (b) Shannon diversity indices of gut microbiota were compared among donor, carriers before FMT, and carriers after FMT. (c) Changes in phylum composition were compared in each carrier according to sampling times. (d) Changes in detected CRE genera, Klebsiella and Escherichia, were compared in each carrier according to sampling times. ${ }^{* *} p<0.001,{ }^{*} p<0.05$. CRE, carbapenem-resistant Enterobacteriaceae; FMT, fecal microbiota transplantation; NMDS, non-metric multidimensional scaling.

\subsection{Comparison of Gut Microbiota between Early Decolonization Carriers and Late Decolonization Carriers}

As four carriers (no. 1, 2, 5, and 10) showed decolonization of CP-CRE within 4 weeks, we compared the gut microbiota between these early decolonization carriers (EDC) and late decolonization carriers (LDC; no. 3, 4, 6, 8, and 9) in terms of times (Figure 2). Although the diversity of gut microbiota was significantly increased in both groups $(p<0.05)$, the increased diversity was higher in the EDC group in terms of time after FMT $\left(\mathrm{R}^{2}=0.271\right)$ than in the LDC group $\left(\mathrm{R}^{2}=0.164\right)$. Bacterial 16S rRNA gene copies increased after FMT; however, the changes in both groups were not statistically significant $(p>0.05)$. In the EDC group, the convergence of recipient gut microbiota to donor microbiota was found within 4 weeks. The dissimilarity of gut microbiota between recipients and donors decreased within 4 weeks after FMT ( $p<0.001$ compared to before FMT). However, the shifts of gut microbiota in the LDC group varied, and convergence to donor microbiota was not found within 4 weeks. The dissimilarity between gut microbiota in LDC carriers and donors also 
decreased $(p<0.01)$; however, the distance was higher than that of the EDC group after 4 weeks. Although the distances of gut microbiota between carriers in the LDC group and the donor were higher than those in the EDC group, the distances also decreased after 9 weeks in the LDC group ( $p<0.01$ after 18 weeks compared to before FMT; Figure S4). Gut microbiota diversity in the LDC group increased after 5 weeks; however, changes in bacterial $16 \mathrm{~S}$ rRNA gene copies were not statistically significant $(p>0.05)$. The relative abundances of Bacteroidetes in the EDC group were higher than those in the LDC group, and the relative abundances of Firmicutes in the EDC group were higher before FMT and lower after FMT than those in the LDC group (Figure S5). Diversity was higher in the EDC group than in the LDC group, before and after FMT treatments. However, these differences were not statistically significant $(p>0.05)$.

(a)

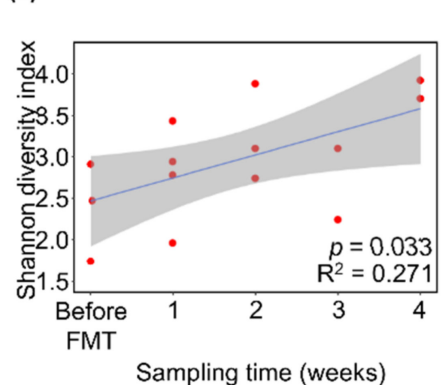

(b)

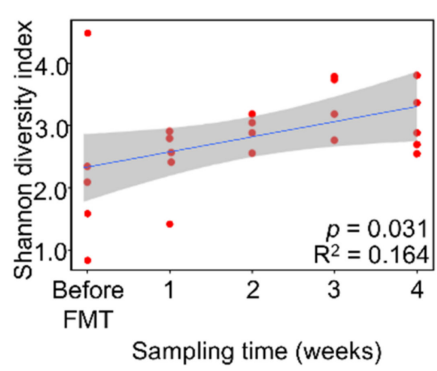

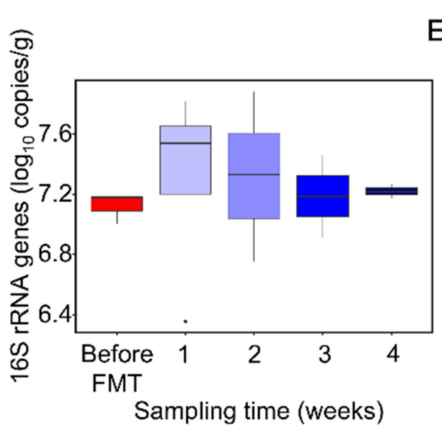

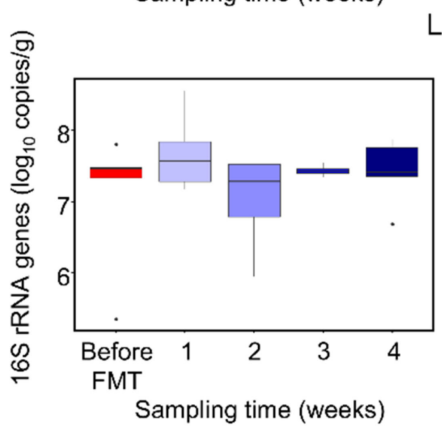

EDC

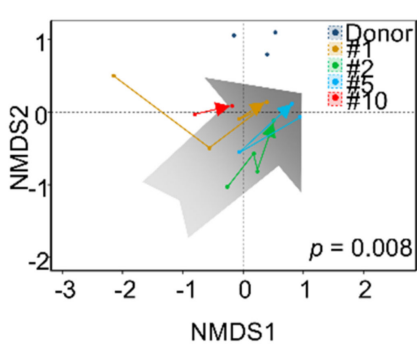

LDC

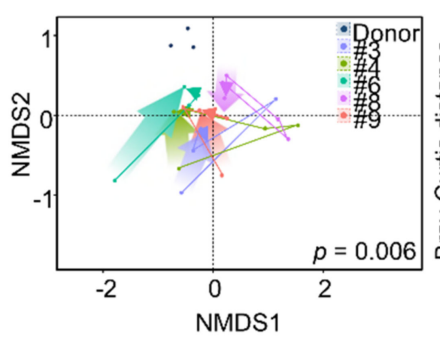

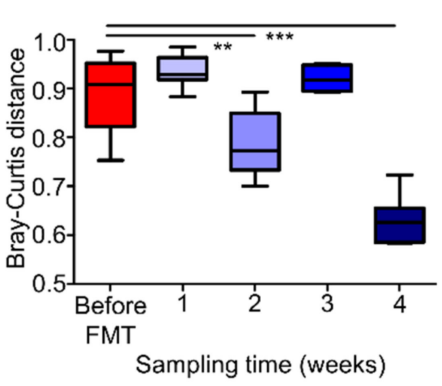

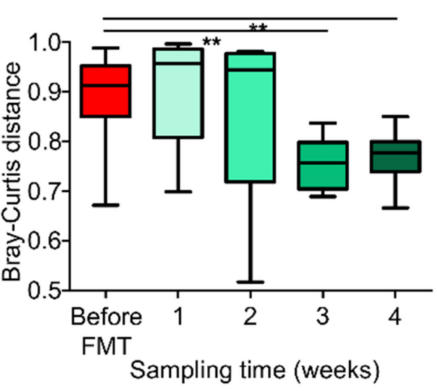

Figure 2. The alterations of gut microbiota were compared between (a) the early decolonization carriers (EDC) and (b) late decolonization carriers (LDC) in terms of sampling times. The changes in diversity were compared using the Shannon diversity index in terms of times. Bacterial $16 \mathrm{~S}$ rRNA gene copies were estimated by quantitative real-time PCR. The convergence of gut microbiota in carriers to donor microbiota was analyzed using NMDS plots. The differences in gut microbiota via times were determined by the Bray-Curtis dissimilarity. ${ }^{* *} p<0.001,{ }^{* *} p<0.01$. NMDS, non-metric multidimensional scaling.

The difference in gut microbiota between the EDC and LDC groups before and within 4 weeks after FMT was analyzed using LEfSe (Figure 3). Frequently detected genera (>1\% of microbiota in each group) were compared between groups. Hungatella was significantly different between the EDC and LDC groups before FMT, whereas 13 genera were different between the two groups after FMT treatments. Hungatella was only detected in the LDC group. The relative abundances of Klebsiella, Corynebacterium, Sellimonas, Dorea, and Clostridium_g7 were significantly higher in the LDC group after FMT than those in the EDC group, whereas relative abundances of Ezakiella, Mogibacterium, uncultured Christensenellaceae (HQ716403_g), uncultured Oscillospiraceae (EU844075_g), Clostridium_g27, Eubacterium, Lactonifactor, and Arcanobacterium were higher in the EDC group compared to the LDC group. The detected CRE genera, Klebsiella and Escherichia, were compared between the EDC and LDC groups according to FMT treatments. The relative abundances of these genera decreased in both groups after FMT. However, the relative abundance of Klebsiella was higher in the LDC group than in the EDC group $(p<0.05$ after FMT), and the 
proportion of Escherichia was lower in the LDC group than in the EDC group before and after FMT.

(a)

$\square$ EDC $\square$ LDC
Before FMT treatment

$\begin{array}{ccccccccc} & & & & & & \text { Hungatella } \\ -6 & -5 & -4 & -3 & -2 & -1 & 0 & 1 & 2 \\ & & & \text { Log10 (LDA score) } & & & \end{array}$

(b)

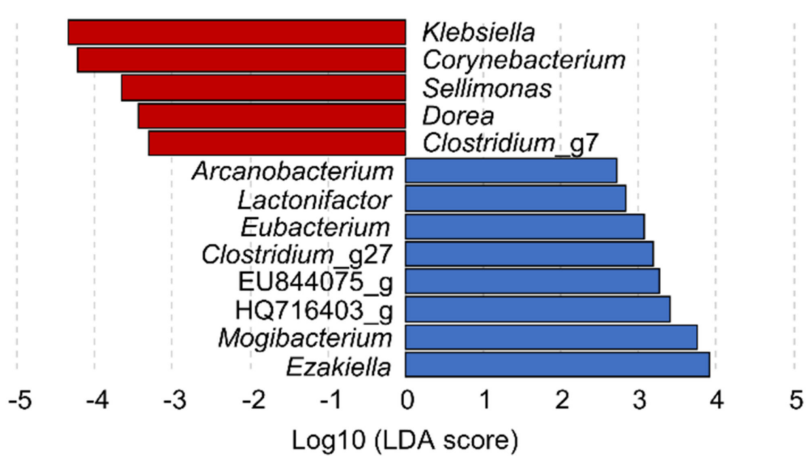

(c)

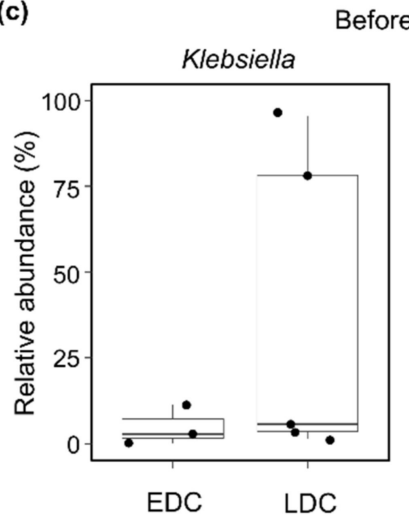

Before FMT treatment

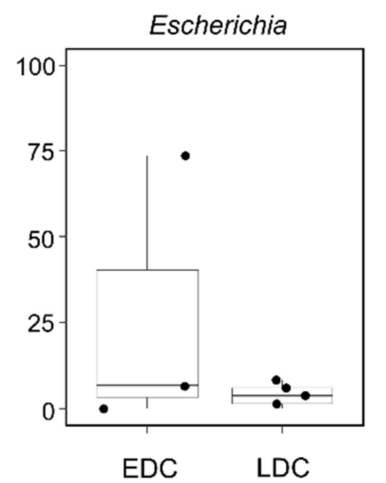

Hungatella

0

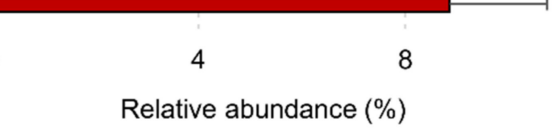

12

After FMT treatment

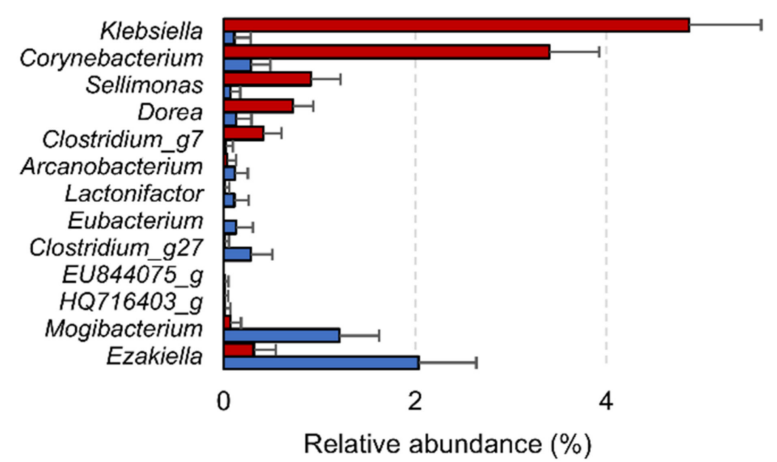

6

Figure 3. Significantly different genera between the EDC and LDC groups (a) before FMT and (b) within 4 weeks after FMT were determined by LEfSe analysis. The relative abundances of different genera were compared between the EDC and LDC groups. Genera with a logarithmic LDA score $>2.0$ were selected as significantly different genera. (c) Relative abundances of Klebsiella and Escherichia were compared between the EDC and LDC groups before and after FMT treatments. ${ }^{*} p<0.05$. EDC, early decolonization; LDC, late decolonization; FMT, fecal microbiota transplantation; LEfSe, linear discriminant analysis effect size; LDA, linear discriminant analysis.

3.4. Different Alteration of Gut Microbiota along Follow-Up Times between EDC and LDC within 4 Weeks after FMT

The alteration in microbiota between the EDC and LDC groups was compared by indicator genera via times (selected by $p<0.05$ ), within 4 weeks after FMT (Figure S6). Three genera (Anaerococcus, Ezakiella, and Peptoniphilus) were significantly changed along times in the EDC group. The relative abundances of these genera increased within 1 week after FMT. In the LDC group, more diverse genera (16 genera) significantly shifted along time after FMT. Klebsiella and Hungatella were significantly detected before FMT, and their proportions increased again after 3 weeks of FMT. The other 14 genera were significantly detected after FMT. Peptoniphilus was a commonly detected genus in both groups after FMT.

Correlations of gut microbiota were compared between the EDC and LDC groups before and after FMT treatments using co-occurrence network analysis (Figure 4). Prior 
to FMT, the keystone genera were different between EDC and LDC. Klebsiella was the common keystone genus in both groups before FMT. However, other keystone genera (Alistipes, Bacteroides, Parabacteroides, Eubacterium_g1 in EDC and Clostridium_g21 in LDC) were different between the two groups before FMT. Within 4 weeks after FMT, Prevotella was a common keystone genus in both groups, whereas Peptoniphilus and Porpyromonas were unique keystone genera in the LDC group. Interactions between genera in the co-occurrence network were more complex in the LDC group than in the EDC group after FMT.

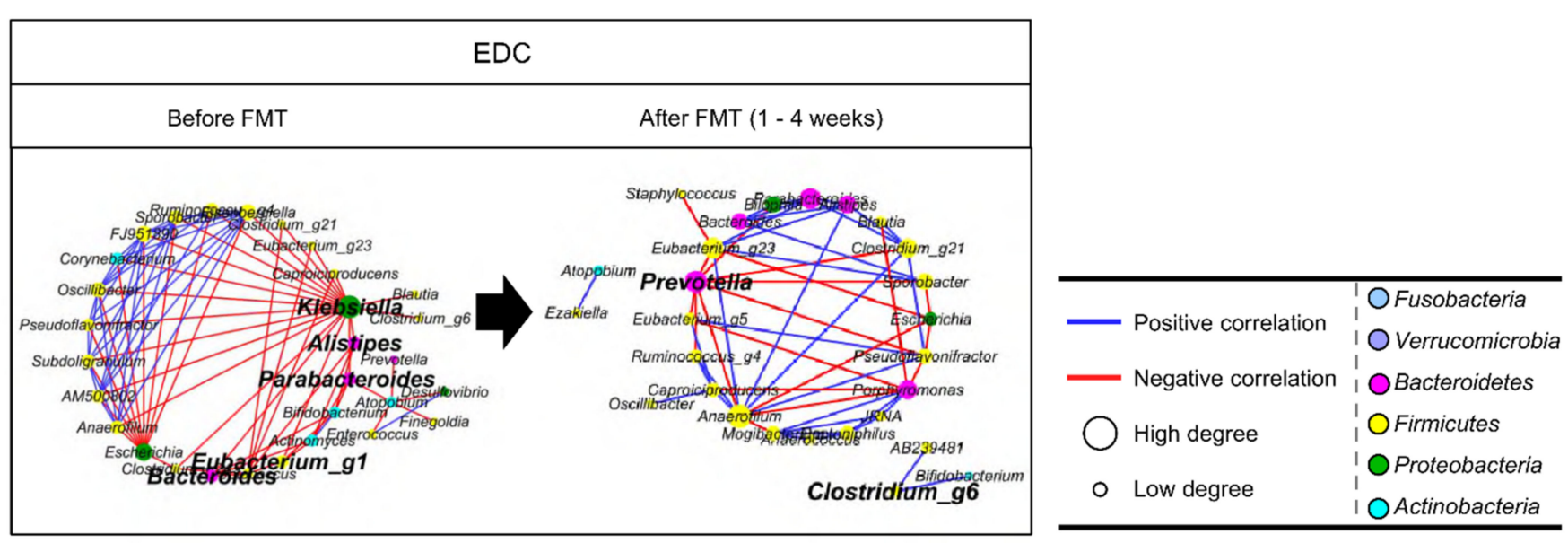

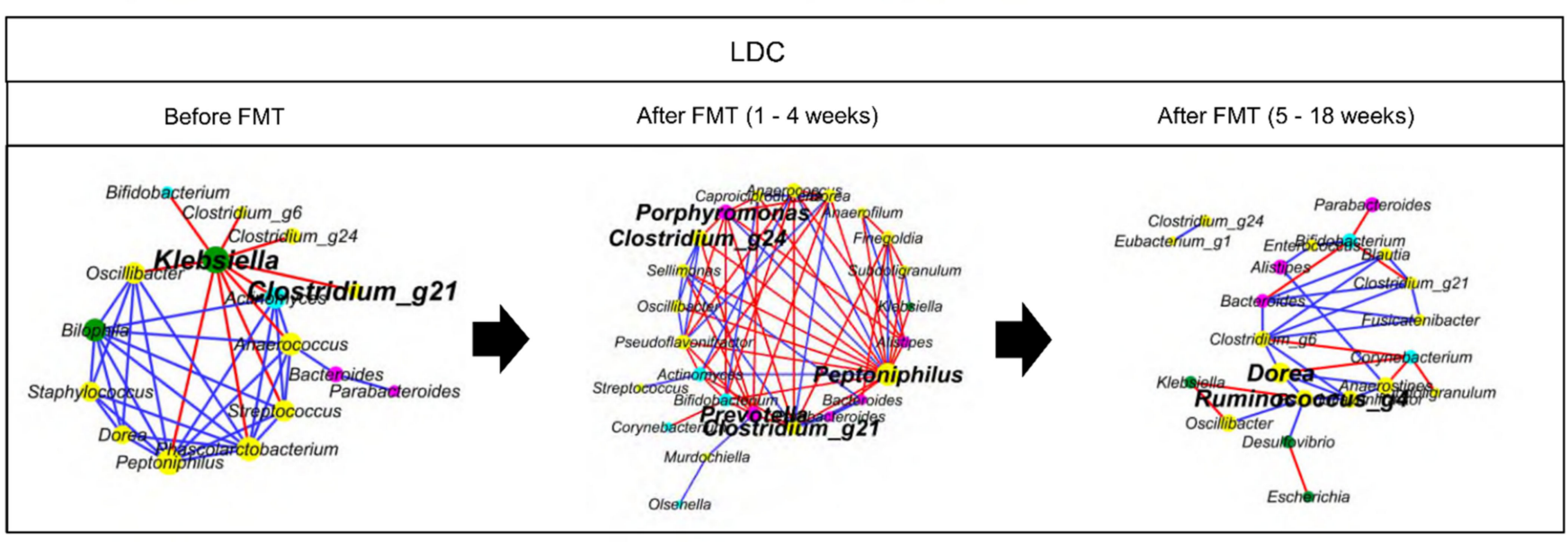

Figure 4. Correlations of genera in gut microbiota were compared between the EDC and LDC groups before and after FMT treatments using co-occurrence network analysis (corrected $p<0.01$ ). Bold letters indicate keystone genera. Keystone genera were selected based on closeness centrality and betweenness centrality values. Circle size indicates the degree. Blue line: positive correlation; red line: negative correlation. EDC, early decolonization; LDC, late decolonization; FMT, fecal microbiota transplantation.

\section{Discussion}

In this study, 90\% (9/10) of CRE carriers showed CRE decolonization within 5 months after FMT treatments, and 40\% (4/10) of carriers showed earlier decolonization (within 1 month after FMT) than other carriers regardless of the origin of donor feces (Table S2). The composition and diversity of microbiota significantly changed and the relative abundance of Bacteroidetes significantly increased after FMT, with decreasing proportions of CRE genera. However, alterations in gut microbiota were different between the EDC and LDC groups. The convergence of microbiota in carriers to donor was detected in the EDC group within 4 weeks, and different keystone genera were found before and after FMT between groups. The relative abundance of Klebsiella was higher in the LDC group than in the EDC group before and after FMT. 
The diversity of gut microbiota significantly increased, and microbiota significantly changed, after FMT. These results are in agreement with previous studies that have shown lower diversity in CRE carriers than in healthy individuals, and increased diversity after FMT treatments [12,28]. Although the relative abundances of Klebsiella and Escherichia were variable among carriers, their enrichment was found in gut microbiota before FMT. As CRE carriers received antibiotic treatment, this treatment could be a reason for the enrichment of these genera as reported in a previous study, which suggested that antibiotics promote intestinal colonization by Enterobacteriaceae [29]. However, their relative abundances decreased, and the Bacteroidetes significantly increased in most carriers, after FMT in the present study. This indicates that FMT can be an alternative option for the decolonization of CRE based on gut microbiota modulation.

Although gut microbiota significantly changed in all carriers after FMT, their alterations were different between the EDC and LDC groups. Previous studies reported the convergence of recipient microbiota towards donor microbiota following FMT treatment $[30,31]$. Notably, we observed that the convergence towards donor microbiota was found in EDC within 4 weeks. This could be due to the gut microbiota of the donor or the difference in indigenous microbiota between the EDC and LDC groups. Although the EDC and LDC were not categorized by the origin of the donor feces, the efficiency of decolonization was different according to donor (Table S2). This suggest that the donor microbiota could partially influence the decolonization of CRE [32]. Hungatella was significantly higher in LDC than in EDC prior to FMT, and five genera were higher in LDC after FMT. Eight genera, including Ezakiella and Mogibacterium, were higher in the EDC group than in the LDC group after FMT. Although these genera were reported as ARB [33-35], significantly abundant genera in the EDC group could have positive effects on gut environment. The loss of Ezakiella was detected in sepsis patients [36]; Mogibacterium was positively correlated with the short-chain fatty acids concentrations [37]; Eubacterium can utilize acetate and lactate to produce butyrate and propionate in the intestine [38]. In particular, the relative abundance of Klebsiella was higher in the LDC group before and after FMT, whereas Escherichia was higher in the EDC group. Although the relative abundances of Klebsiella decreased 1-2 weeks after FMT in the LDC group, the proportion increased again after 3-4 weeks. Therefore, CRE carriers in the LDC group did not decolonize within 4 weeks. This indicated that the high abundance of Klebsiella could be related to the late decolonization or the necessity of repeated FMT for CRE decolonization.

Co-occurrence network analysis showed that different gut microbiota between groups before FMT could affect the alteration of microbiota after FMT. Klebsiella was a common keystone genus in both groups before FMT; however, more genera negatively correlated with Klebsiella in EDC than LDC. In particular, the genera within Bacteroidetes (Alistipes, Bacteroides, and Parabacteroides), which were keystone genera in EDC before FMT, negatively correlated with Klebsiella. However, the keystone genus (Clostridium) within Firmicutes negatively correlated with Klebsiella in the LDC group. Keystone genera are important for maintaining the structure, diversity, and function of the ecosystem through biotic interactions with other members [39]. Bacteroides spp. demonstrated that they have the capacity to decompose polysaccharides and produce beneficial metabolites [40]. In addition, Bacteroidetes can prevent the colonization of K. pneumoniae in the gut by fortifying the gut immune barrier via IL-36 and macrophages [41]. These results indicated that the gut microbiota was different between the EDC and LDC groups prior to FMT, and this difference could affect the timing of decolonization after FMT. Although Prevotella was a common keystone genus in both groups within 4 weeks after FMT, more complex interactions and more keystone genera were detected in the LDC group compared to the EDC group. In a previous study, Prevotellaceae was reported as a faster colonizer after FMT, and the colonization of Prevotella spp. could induce metabolic changes in microbiota, which reduced IL-18 production and consequently exacerbated intestinal inflammation [42,43]. Thus, Prevotella was detected as a keystone genus after FMT in both groups, and a significantly shifted genus within 2 weeks after FMT. 
FMT treatment has been increasingly used for various disorders, including CDI, ulcerative colitis, and Crohn's disease. The therapeutic effect of FMT demonstrated the expansion of donor microbiota and defect correction in microbiota composition $[44,45]$. Various factors, including bacteria, bacteriophages, metabolites, and extracellular vesicles, in donor feces could influence the modulation of the gut microbiota and the decolonization of CRE in recipients [46-51]. Previous studies have reported the decolonization of ARB, including CRE, ESBL-E, and VRE, using FMT [7,52-54]. However, most reports demonstrated that ARB disappeared after FMT for recurrent CDI patients. A few studies focused on the decolonization of CRE in carriers, regardless of CDI, by FMT. The present study has the potential to extend our understanding of ARB by FMT.

The limitations of this study were the relatively small number of carriers and the need to detail microbiota shifts based on antibiotic resistance genes. However, this study analyzed the longitudinal shifts of microbiota after FMT and used CRE carriers regardless of CDI. Further, studies are necessary to analyze the modulation of the gut microbiome ecosystem using whole-metagenomics, metatranscriptomics and metabolomics.

\section{Conclusions}

FMT treatments for CRE carriers showed $90 \%$ decolonization in this study. The decolonization period was different for the gut microbiota of carriers before FMT. Genera within Bacteroidetes were keystones in gut microbiota of the EDC group before FMT. The relative abundance of Klebsiella was lower in the EDC group than in the LDC group, before and after FMT. These results could be applied to predict the decolonization period after FMT and determine the necessity of repeated FMTs for the decolonization of ARB.

Supplementary Materials: The following are available online at https:/ /www.mdpi.com/2076-260 7/9/2/352/s1, Table S1: Detection of carbapenem-resistant Enterobacteriaceae (CRE) in carriers by culture assays during fecal microbiota transplantation (FMT) treatment, Table S2: The origin of donor feces used for fecal microbiota transplantation (FMT) in each carrier, Figure S1: Sampling times and decolonization day for each carrier, Figure S2: The effects of influencing factors on gut microbiota difference, Figure S3: Changes in genus composition during fecal microbiota transplantation (FMT) treatment in each carrier, Figure S4: Alterations of gut microbiota in the late decolonization carriers (LDC) group after 5 weeks, Figure S5: Relative abundances of Bacteroidetes and Firmicutes and microbiota diversity were compared between the early decolonization carriers (EDC) and late decolonization carriers (LDC) groups before and after fecal microbiota transplantation (FMT) treatment, Figure S6: Indicator genera in terms of times were compared between the early decolonization carriers (EDC) and late decolonization carriers (LDC) groups.

Author Contributions: Conceptualization, S.S.L. and B.-S.K.; methodology, D.Y., S.S.L. and B.-S.K.; validation, S.S.L. and B.-S.K.; formal analysis, J.-J.L. and S.S.L.; investigation, J.-J.L., D.Y., K.T.S., D.J.K., H.-J.W. and S.S.L.; resources, D.Y., K.T.S., D.J.K., H.-J.W. and S.S.L.; data curation, J.-J.L. and B.-S.K.; writing—original draft preparation, J.-J.L. and D.Y.; writing—review and editing, K.T.S., D.J.K., S.S.L. and B.-S.K.; visualization, J.-J.L.; supervision, S.S.L. and B.-S.K.; project administration, S.S.L. and B.-S.K.; funding acquisition, K.T.S., D.J.K., S.S.L. and B.-S.K. All authors have read and agreed to the published version of the manuscript.

Funding: This research was funded by Basic Science Research Program (2019R111A3A01060465 and 2020R1A6A1A03043026) through the National Research Foundation of Korea (NRF) funded by the Ministry of Education, Bio \& Medical Technology Development Program through the National Research Foundation of Korea (NRF) funded by the Ministry of Science, ICT \& Future Planning (NRF-2017M3A9F3043837).

Institutional Review Board Statement: The study was conducted according to the guidelines of the Declaration of Helsinki, and approved by the Severance Hospital Institutional Review Board, Seoul, Korea (IRB no. 4-2016-0871) and the Institutional Review Board at Hallym University Chuncheon Sacred Heart Hospital (IRB no. 2019-05-012 and 2020-08-006). This study was registered on the ClinicalTrials.gov public website (no. NCT04583098). 
Informed Consent Statement: Informed consent was obtained from all subjects involved in the study. Written informed consent has been obtained from the patients to publish this paper.

Data Availability Statement: The sequence reads obtained from this study are available in the EMBL SRA database under the study number PRJEB41057 (http:/ / ebi.ac.uk/ena/data/view /PRJEB41057).

Conflicts of Interest: The authors declare no conflict of interest. The funders had no role in the design of the study; in the collection, analyses, or interpretation of data; in the writing of the manuscript, or in the decision to publish the results.

\section{References}

1. Bonomo, R.A.; Burd, E.M.; Conly, J.; Limbago, B.M.; Poirel, L.; Segre, J.A.; Westblade, L.F. Carbapenemase-Producing Organisms: A Global Scourge. Clin. Infect. Dis. 2018, 66, 1290-1297. [CrossRef] [PubMed]

2. Doi, Y.; Paterson, D.L. Carbapenemase-producing Enterobacteriaceae. Semin. Respir. Crit. Care Med. 2015, 36, 74-84. [CrossRef]

3. Bar-Yoseph, H.; Hussein, K.; Braun, E.; Paul, M. Natural history and decolonization strategies for ESBL/carbapenem-resistant Enterobacteriaceae carriage: Systematic review and meta-analysis. J. Antimicrob. Chemother. 2016, 71, 2729-2739. [CrossRef] [PubMed]

4. Campos, A.C.; Albiero, J.; Ecker, A.B.; Kuroda, C.M.; Meirelles, L.E.; Polato, A.; Tognim, M.C.; Wingeter, M.A.; Teixeira, J.J. Outbreak of Klebsiella pneumoniae carbapenemase-producing K pneumoniae: A systematic review. Am. J. Infect. Control 2016, 44, 1374-1380. [CrossRef]

5. Buffie, C.G.; Jarchum, I.; Equinda, M.; Lipuma, L.; Gobourne, A.; Viale, A.; Ubeda, C.; Xavier, J.; Pamer, E.G. Profound alterations of intestinal microbiota following a single dose of clindamycin results in sustained susceptibility to Clostridium difficile-induced colitis. Infect. Immun. 2012, 80, 62-73. [CrossRef] [PubMed]

6. Debast, S.B.; Bauer, M.P.; Kuijper, E.J.; European Society of Clinical, M.; Infectious, D. European Society of Clinical Microbiology and Infectious Diseases: Update of the treatment guidance document for Clostridium difficile infection. Clin. Microbiol. Infect. 2014, 20 (Suppl. S2), 1-26. [CrossRef]

7. Millan, B.; Park, H.; Hotte, N.; Mathieu, O.; Burguiere, P.; Tompkins, T.A.; Kao, D.; Madsen, K.L. Fecal Microbial Transplants Reduce Antibiotic-resistant Genes in Patients with Recurrent Clostridium difficile Infection. Clin. Infect. Dis. 2016, 62, 1479-1486. [CrossRef] [PubMed]

8. Rossen, N.G.; Fuentes, S.; van der Spek, M.J.; Tijssen, J.G.; Hartman, J.H.; Duflou, A.; Lowenberg, M.; van den Brink, G.R.; Mathus-Vliegen, E.M.; de Vos, W.M.; et al. Findings From a Randomized Controlled Trial of Fecal Transplantation for Patients with Ulcerative Colitis. Gastroenterology 2015, 149, 110-118.e114. [CrossRef]

9. Suskind, D.L.; Brittnacher, M.J.; Wahbeh, G.; Shaffer, M.L.; Hayden, H.S.; Qin, X.; Singh, N.; Damman, C.J.; Hager, K.R.; Nielson, H.; et al. Fecal microbial transplant effect on clinical outcomes and fecal microbiome in active Crohn's disease. Inflamm. Bowel Dis. 2015, 21, 556-563. [CrossRef]

10. Saha, S.; Tariq, R.; Tosh, P.K.; Pardi, D.S.; Khanna, S. Faecal microbiota transplantation for eradicating carriage of multidrugresistant organisms: A systematic review. Clin. Microbiol. Infect. 2019, 25, 958-963. [CrossRef]

11. Manges, A.R.; Steiner, T.S.; Wright, A.J. Fecal microbiota transplantation for the intestinal decolonization of extensively antimicrobial-resistant opportunistic pathogens: A review. Infect. Dis. 2016, 48, 587-592. [CrossRef] [PubMed]

12. Korach-Rechtman, H.; Hreish, M.; Fried, C.; Gerassy-Vainberg, S.; Azzam, Z.S.; Kashi, Y.; Berger, G. Intestinal Dysbiosis in Carriers of Carbapenem-Resistant Enterobacteriaceae. mSphere 2020, 5, e00173-20. [CrossRef]

13. Kim, Y.K.; Song, S.A.; Lee, J.N.; Oh, M.; Jo, K.M.; Kim, H.J.; Lee, J.H.; Park, J.; Jang, H.J.; Kim, H.K.; et al. Clinical factors predicting persistent carriage of Klebsiella pneumoniae carbapenemase-producing carbapenem-resistant Enterobacteriaceae among patients with known carriage. J. Hosp. Infect. 2018, 99, 405-412. [CrossRef]

14. Cammarota, G.; Ianiro, G.; Tilg, H.; Rajilic-Stojanovic, M.; Kump, P.; Satokari, R.; Sokol, H.; Arkkila, P.; Pintus, C.; Hart, A.; et al. European consensus conference on faecal microbiota transplantation in clinical practice. Gut 2017, 66, 569-580. [CrossRef] [PubMed]

15. Costello, S.P.; Tucker, E.C.; La Brooy, J.; Schoeman, M.N.; Andrews, J.M. Establishing a Fecal Microbiota Transplant Service for the Treatment of Clostridium difficile Infection. Clin. Infect. Dis. 2016, 62, 908-914. [CrossRef]

16. Terveer, E.M.; van Beurden, Y.H.; Goorhuis, A.; Seegers, J.; Bauer, M.P.; van Nood, E.; Dijkgraaf, M.G.W.; Mulder, C.J.J.; Vandenbroucke-Grauls, C.; Verspaget, H.W.; et al. How to: Establish and run a stool bank. Clin. Microbiol. Infect. 2017, 23, 924-930. [CrossRef]

17. Division of Human Blood Safety Surveillance. Transfusion Guideline, 3rd ed.; Korean Centers for Disease Control and Prevention: Cheongju, Korea, 2013.

18. Clinical \& Laboratory Standards Institute. Performance Standards for Antimicrobial Susceptibility Testing. M100S; CLSI: Wayne, PA, USA, 2016.

19. Morey, K.E.; Vega, R.; Cassidy, P.M.; Buser, G.L.; Rayar, J.K.; Myers, J.A.; Weissman, S.J.; Beldavs, Z.G.; Pfeiffer, C.D. Evaluation of the Carba NP Test in Oregon, 2013. Antimicrob. Agents Chemother. 2017, 61, e3005-15. [CrossRef] [PubMed] 
20. Jeong, S.H.; Kim, H.S.; Kim, J.S.; Shin, D.H.; Kim, H.S.; Park, M.J.; Shin, S.; Hong, J.S.; Lee, S.S.; Song, W. Prevalence and Molecular Characteristics of Carbapenemase-Producing Enterobacteriaceae from Five Hospitals in Korea. Ann. Lab. Med. 2016, 36, 529-535. [CrossRef] [PubMed]

21. Kim, H.E.; Lee, J.J.; Lee, M.J.; Kim, B.S. Analysis of microbiome in raw chicken meat from butcher shops and packaged products in South Korea to detect the potential risk of foodborne illness. Food Res. Int. 2019, 122, 517-527. [CrossRef] [PubMed]

22. Park, J.U.; Oh, B.; Lee, J.P.; Choi, M.H.; Lee, M.J.; Kim, B.S. Influence of Microbiota on Diabetic Foot Wound in Comparison with Adjacent Normal Skin Based on the Clinical Features. BioMed Res. Int. 2019, 2019, 7459236. [CrossRef]

23. Callahan, B.J.; McMurdie, P.J.; Rosen, M.J.; Han, A.W.; Johnson, A.J.; Holmes, S.P. DADA2: High-resolution sample inference from Illumina amplicon data. Nat. Methods 2016, 13, 581-583. [CrossRef] [PubMed]

24. Yoon, S.H.; Ha, S.M.; Kwon, S.; Lim, J.; Kim, Y.; Seo, H.; Chun, J. Introducing EzBioCloud: A taxonomically united database of 16S rRNA gene sequences and whole-genome assemblies. Int. J. Syst. Evol. Microbiol. 2017, 67, 1613-1617. [CrossRef]

25. Schloss, P.D.; Westcott, S.L.; Ryabin, T.; Hall, J.R.; Hartmann, M.; Hollister, E.B.; Lesniewski, R.A.; Oakley, B.B.; Parks, D.H.; Robinson, C.J.; et al. Introducing mothur: Open-source, platform-independent, community-supported software for describing and comparing microbial communities. Appl. Environ. Microbiol. 2009, 75, 7537-7541. [CrossRef] [PubMed]

26. Segata, N.; Izard, J.; Waldron, L.; Gevers, D.; Miropolsky, L.; Garrett, W.S.; Huttenhower, C. Metagenomic biomarker discovery and explanation. Genome Biol. 2011, 12, R60. [CrossRef] [PubMed]

27. Faust, K.; Raes, J. CoNet app: Inference of biological association networks using Cytoscape. F1000Research 2016, 5, 1519. [CrossRef] [PubMed]

28. Staley, C.; Kaiser, T.; Vaughn, B.P.; Graiziger, C.T.; Hamilton, M.J.; Rehman, T.U.; Song, K.; Khoruts, A.; Sadowsky, M.J. Predicting recurrence of Clostridium difficile infection following encapsulated fecal microbiota transplantation. Microbiome 2018, 6, 166. [CrossRef] [PubMed]

29. Ubeda, C.; Taur, Y.; Jenq, R.R.; Equinda, M.J.; Son, T.; Samstein, M.; Viale, A.; Socci, N.D.; van den Brink, M.R.; Kamboj, M.; et al. Vancomycin-resistant Enterococcus domination of intestinal microbiota is enabled by antibiotic treatment in mice and precedes bloodstream invasion in humans. J. Clin. Investig. 2010, 120, 4332-4341. [CrossRef]

30. Seekatz, A.M.; Aas, J.; Gessert, C.E.; Rubin, T.A.; Saman, D.M.; Bakken, J.S.; Young, V.B. Recovery of the gut microbiome following fecal microbiota transplantation. mBio 2014, 5, e00893-14. [CrossRef]

31. Weingarden, A.; Gonzalez, A.; Vazquez-Baeza, Y.; Weiss, S.; Humphry, G.; Berg-Lyons, D.; Knights, D.; Unno, T.; Bobr, A.; Kang, J.; et al. Dynamic changes in short- and long-term bacterial composition following fecal microbiota transplantation for recurrent Clostridium difficile infection. Microbiome 2015, 3, 10. [CrossRef] [PubMed]

32. Wilson, B.C.; Vatanen, T.; Cutfield, W.S.; O'Sullivan, J.M. The Super-Donor Phenomenon in Fecal Microbiota Transplantation. Front. Cell. Infect. Microbiol. 2019, 9, 2. [CrossRef]

33. Liu, X.; Zheng, H.; Lu, R.; Huang, H.; Zhu, H.; Yin, C.; Mo, Y.; Wu, J.; Liu, X.; Deng, M.; et al. Intervening Effects of Total Alkaloids of Corydalis saxicola Bunting on Rats with Antibiotic-Induced Gut Microbiota Dysbiosis Based on 16S rRNA Gene Sequencing and Untargeted Metabolomics Analyses. Front. Microbiol. 2019, 10, 1151. [CrossRef]

34. Dragomirescu, C.C.; Lixandru, B.E.; Coldea, I.L.; Corneli, O.N.; Pana, M.; Palade, A.M.; Cristea, V.C.; Suciu, I.; Suciu, G.; Manolescu, L.S.C.; et al. Antimicrobial Susceptibility Testing for Corynebacterium Species Isolated from Clinical Samples in Romania. Antibiotics 2020, 9, 31. [CrossRef] [PubMed]

35. Pettigrew, M.M.; Gent, J.F.; Kong, Y.; Halpin, A.L.; Pineles, L.; Harris, A.D.; Johnson, J.K. Gastrointestinal Microbiota Disruption and Risk of Colonization with Carbapenem-resistant Pseudomonas aeruginosa in Intensive Care Unit Patients. Clin. Infect. Dis. 2019, 69, 604-613. [CrossRef] [PubMed]

36. Agudelo-Ochoa, G.M.; Valdes-Duque, B.E.; Giraldo-Giraldo, N.A.; Jaillier-Ramirez, A.M.; Giraldo-Villa, A.; Acevedo-Castano, I.; Yepes-Molina, M.A.; Barbosa-Barbosa, J.; Benitez-Paez, A. Gut microbiota profiles in critically ill patients, potential biomarkers and risk variables for sepsis. Gut Microbes 2020, 12, 1707610. [CrossRef]

37. Zhu, L.; Liao, R.; Tu, W.; Lu, Y.; Cai, X. Pyrodextrin enhances intestinal function through changing the intestinal microbiota composition and metabolism in early weaned piglets. Appl. Microbiol. Biotechnol. 2020, 104, 4141-4154. [CrossRef]

38. Engels, C.; Ruscheweyh, H.J.; Beerenwinkel, N.; Lacroix, C.; Schwab, C. The Common Gut Microbe Eubacterium hallii also Contributes to Intestinal Propionate Formation. Front. Microbiol. 2016, 7, 713. [CrossRef]

39. Trosvik, P.; de Muinck, E.J. Ecology of bacteria in the human gastrointestinal tract-identification of keystone and foundation taxa. Microbiome 2015, 3, 44. [CrossRef]

40. Comstock, L.E. Importance of glycans to the host-bacteroides mutualism in the mammalian intestine. Cell Host Microbe 2009, 5, 522-526. [CrossRef]

41. Sequeira, R.P.; McDonald, J.A.K.; Marchesi, J.R.; Clarke, T.B. Commensal Bacteroidetes protect against Klebsiella pneumoniae colonization and transmission through IL-36 signalling. Nat. Microbiol. 2020, 5, 304-313. [CrossRef] [PubMed]

42. Goloshchapov, O.V.; Olekhnovich, E.I.; Sidorenko, S.V.; Moiseev, I.S.; Kucher, M.A.; Fedorov, D.E.; Pavlenko, A.V.; Manolov, A.I.; Gostev, V.V.; Veselovsky, V.A.; et al. Long-term impact of fecal transplantation in healthy volunteers. BMC Microbiol. $2019,19,312$. [CrossRef]

43. Iljazovic, A.; Roy, U.; Galvez, E.J.C.; Lesker, T.R.; Zhao, B.; Gronow, A.; Amend, L.; Will, S.E.; Hofmann, J.D.; Pils, M.C.; et al. Perturbation of the gut microbiome by Prevotella spp. enhances host susceptibility to mucosal inflammation. Mucosal. Immunol. 2020. [CrossRef] [PubMed] 
44. Lopez, J.; Grinspan, A. Fecal Microbiota Transplantation for Inflammatory Bowel Disease. Gastroenterol. Hepatol. $2016,12,374-379$.

45. Leszczyszyn, J.J.; Radomski, M.; Leszczyszyn, A.M. Intestinal microbiota transplant-Current state of knowledge. Reumatologia 2016, 54, 24-28. [CrossRef] [PubMed]

46. Umu, O.C.; Bauerl, C.; Oostindjer, M.; Pope, P.B.; Hernandez, P.E.; Perez-Martinez, G.; Diep, D.B. The Potential of Class II Bacteriocins to Modify Gut Microbiota to Improve Host Health. PLoS ONE 2016, 11, e0164036. [CrossRef] [PubMed]

47. Andoh, A.; Tsujikawa, T.; Hata, K.; Araki, Y.; Kitoh, K.; Sasaki, M.; Yoshida, T.; Fujiyama, Y. Elevated circulating platelet-derived microparticles in patients with active inflammatory bowel disease. Am. J. Gastroenterol. 2005, 100, 2042-2048. [CrossRef]

48. Leonetti, D.; Reimund, J.M.; Tesse, A.; Viennot, S.; Martinez, M.C.; Bretagne, A.L.; Andriantsitohaina, R. Circulating microparticles from Crohn's disease patients cause endothelial and vascular dysfunctions. PLoS ONE 2013, 8, e73088. [CrossRef]

49. Sweere, J.M.; Van Belleghem, J.D.; Ishak, H.; Bach, M.S.; Popescu, M.; Sunkari, V.; Kaber, G.; Manasherob, R.; Suh, G.A.; Cao, X.; et al. Bacteriophage trigger antiviral immunity and prevent clearance of bacterial infection. Science 2019, 363. [CrossRef]

50. Rasmussen, T.S.; Mentzel, C.M.J.; Kot, W.; Castro-Mejia, J.L.; Zuffa, S.; Swann, J.R.; Hansen, L.H.; Vogensen, F.K.; Hansen, A.K.; Nielsen, D.S. Faecal virome transplantation decreases symptoms of type 2 diabetes and obesity in a murine model. Gut 2020, 69, 2122-2130. [CrossRef]

51. Duan, Y.; Llorente, C.; Lang, S.; Brandl, K.; Chu, H.; Jiang, L.; White, R.C.; Clarke, T.H.; Nguyen, K.; Torralba, M.; et al. Bacteriophage targeting of gut bacterium attenuates alcoholic liver disease. Nature 2019, 575, 505-511. [CrossRef]

52. Wong, W.F.; Santiago, M. Microbial approaches for targeting antibiotic-resistant bacteria. Microb. Biotechnol. 2017, 10, 1047-1053. [CrossRef]

53. Jouhten, H.; Mattila, E.; Arkkila, P.; Satokari, R. Reduction of Antibiotic Resistance Genes in Intestinal Microbiota of Patients with Recurrent Clostridium difficile Infection after Fecal Microbiota Transplantation. Clin. Infect. Dis. 2016, 63, 710-711. [CrossRef] [PubMed]

54. Bilinski, J.; Grzesiowski, P.; Sorensen, N.; Madry, K.; Muszynski, J.; Robak, K.; Wroblewska, M.; Dzieciatkowski, T.; Dulny, G.; Dwilewicz-Trojaczek, J.; et al. Fecal Microbiota Transplantation in Patients with Blood Disorders Inhibits Gut Colonization with Antibiotic-Resistant Bacteria: Results of a Prospective, Single-Center Study. Clin. Infect. Dis. 2017, 65, 364-370. [CrossRef] [PubMed] 